\title{
Generalized $\oplus$-Radical Supplemented Modules
}

\author{
Burcu Nişancı Türkmen ${ }^{1}$ and Ali Pancar ${ }^{2}$ \\ ${ }^{1}$ Faculty of Art and Science, Amasya University, İpekköy, 05100 Amasya, Turkey \\ ${ }^{2}$ Department of Mathematics, Faculty of Art and Science, Ondokuz Mayis University, Kurupelit, 55139 Samsun, Turkey
}

Correspondence should be addressed to Burcu Nişancı Türkmen; burcunisancie@hotmail.com

Received 28 October 2013; Accepted 16 December 2013; Published 14 January 2014

Academic Editors: A. V. Kelarev and S. Yang

Copyright (C) 2014 B. Nişancı Türkmen and A. Pancar. This is an open access article distributed under the Creative Commons Attribution License, which permits unrestricted use, distribution, and reproduction in any medium, provided the original work is properly cited.

\begin{abstract}
Çalışııı and Türkmen called a module $M$ generalized $\oplus$-supplemented if every submodule has a generalized supplement that is a direct summand of $M$. Motivated by this, it is natural to introduce another notion that we called generalized $\oplus$-radical supplemented modules as a proper generalization of generalized $\oplus$-supplemented modules. In this paper, we obtain various properties of generalized $\oplus$-radical supplemented modules. We show that the class of generalized $\oplus$-radical supplemented modules is closed under finite direct sums. We attain that over a Dedekind domain a module $M$ is generalized $\oplus$-radical supplemented if and only if $M / P(M)$ is generalized $\oplus$-radical supplemented. We completely determine the structure of these modules over left $V$-rings. Moreover, we characterize semiperfect rings via generalized $\oplus$-radical supplemented modules.
\end{abstract}

\section{Introduction}

Throughout the whole text, all rings are to be associative; unit and all modules are left unitary. We specially mention [1-4] among books concerning the structures of modules and rings. We shall write $N \leq M(N \ll M)$ if $N$ is a submodule of $M($ small in $M)$. By $\operatorname{Rad}(M)$, we denote the radical of $M$. Let $U, V \leq M$. $V$ is called a supplement of $U$ in $M$ if it is minimal with respect to $M=U+V$. $V$ is a supplement of $U$ in $M$ if and only if $M=U+V$ and $U \cap V \ll V$ [4]. A module $M$ is called supplemented if every submodule of $M$ has a supplement, and it is called $\oplus$ supplemented if every submodule of $M$ has a supplement that is a direct summand of $M$ [5]. Clearly every $\oplus$-supplemented module is supplemented. In [6], Zöschinger introduced a notion of modules whose radical has supplements called radical supplemented. Xue defined generalized supplemented modules as another generalization of supplemented modules [7]. Let $U$ be any submodule of $M$. If there exists a submodule $V$ of $M$ such that $M=U+V$ and $U \cap V \subseteq \operatorname{Rad}(V), V$ is called a generalized supplement of $U$ in $M . M$ is called generalized supplemented if every submodule of $M$ has a generalized supplement in $M$. Also Çalışıcı and Türkmen called a module $M$ generalized $\oplus$-supplemented if every submodule has a generalized supplement that is a direct summand of $M$ as a generalization of $\oplus$-supplemented modules [8]. So it is natural to introduce another notion that we called generalized $\oplus$-radical supplemented modules. A module $M$ is called generalized $\oplus$-radical supplemented if every submodule containing radical has a generalized supplement that is a direct summand of $M$.

In this paper we obtain various properties of generalized $\oplus$-radical supplemented modules as a proper generalization of generalized $\oplus$-supplemented modules. We prove the following indications.

(i) Every generalized $\oplus$-radical supplemented module has a radical direct summand.

(ii) $P(M)$ is a generalized $\oplus$-radical supplemented module for every $R$-module $M$.

(iii) The class of generalized $\oplus$-radical supplemented modules is closed under finite direct sums.

(iv) If $M$ is a generalized $\oplus$-radical supplemented module, then $M / U$ is a generalized $\oplus$-radical supplemented module for every fully invariant submodule $U$ of $M$.

(v) Let $\left\{M_{i}\right\}_{i \in I}$ be any infinite collection of generalized $\oplus$ radical supplemented modules, and let $M=\oplus_{i \in I} M_{i}$. 
If $M$ is a duo module, then $M$ is generalized $\oplus$-radical supplemented.

(vi) Over a Dedekind domain a module $M$ is generalized $\oplus$-radical supplemented if and only if $M / P(M)$ is generalized $\oplus$-radical supplemented.

(vii) Every generalized $\oplus$-radical supplemented $R$-module is injective if and only if $R$ is left Noetherian $V$-ring.

(viii) A ring $R$ is semiperfect if and only if every finitely generated free $R$-module is generalized $\oplus$-radical supplemented.

\section{Generalized $\oplus$-Radical Supplemented Modules}

Definition 1. A module $M$ is called generalized $\oplus$-radical supplemented if every submodule containing radical has a generalized supplement that is a direct summand of $M$.

Recall that a module $M$ is called radical if $M$ has no maximal submodules; that is, $\operatorname{Rad}(M)=M$. For a module $M, P(M)$ will indicate the sum of all radical submodules of $M$. If $P(M)=0, M$ is called reduced. Note that $P(M)$ is the largest radical submodule of $M$ [4].

Now we have the following simple fact, which lays a key role in our study.

Lemma 2. Let $M$ be an $R$-module. If $\operatorname{Rad}(M)=M$, then $M$ is a generalized $\oplus$-radical supplemented module.

Proof. Since $\operatorname{Rad}(M)=M, M$ has the trivial generalized supplement 0 in $M$. Consequently, $M$ is a generalized $\oplus$ radical supplemented module.

Corollary 3. $P(M)$ is a generalized $\oplus$-radical supplemented module for every $R$-module $M$.

Proposition 4. Every generalized $\oplus$-radical supplemented module has a radical direct summand.

Proof. Let $M$ be a generalized $\oplus$-radical supplemented module. Then there exist submodules $V$ and $V^{\prime}$ of $M$ such that $M=\operatorname{Rad}(M)+V, \operatorname{Rad}(M) \cap V \subseteq \operatorname{Rad}(V)$, and $M=V \oplus V^{\prime}$. Since $\operatorname{Rad}(M)=\operatorname{Rad}(V) \oplus \operatorname{Rad}\left(V^{\prime}\right)$ by [4, Theorem 21.6], then $M=\operatorname{Rad}\left(V^{\prime}\right)+V$. Applying the modular law, we obtain that $\operatorname{Rad}\left(V^{\prime}\right)=V^{\prime}$.

Recall from [4] that a submodule $U$ of an $R$-module $M$ is called fully invariant if $f(U)$ is contained in $U$ for every $R$ endomorphism $f$ of $M$. A module $M$ is called $d u o$, if every submodule of $M$ is fully invariant [9].

Theorem 5. The following statements hold over a ring $R$.

(1) Let $\left\{M_{i}\right\}_{i \in\{1,2, \ldots, n\}}$ be any finite collection of modules and $M=M_{1} \oplus M_{2} \oplus \cdots \oplus M_{n}$. Then $M$ is generalized $\oplus$-radical supplemented if, for each $1 \leq i \leq n, M_{i}$ is generalized $\oplus$-radical supplemented.
(2) Let $\left\{M_{i}\right\}_{i \in I}$ be any infinite collection of modules and $=$ $\oplus_{i \in I} M_{i}$. If $M$ is a duo module, then $M$ is generalized $\oplus$ radical supplemented if, for each $i \in I, M_{i}$ is generalized $\oplus$-radical supplemented.

Proof. (1) Let $M_{i}$ be a generalized $\oplus$-radical supplemented module for each $1 \leq i \leq n$. To prove that $M$ is a generalized $\oplus$-radical supplemented module it is sufficient by induction on $n$ to prove this is the case when $n=2$. Hence, suppose that $n=2$. Let $N$ be any submodule of $M$ with $\operatorname{Rad}(M) \subseteq N$. Then $M=M_{1}+M_{2}+N$ so that $M_{1}+M_{2}+N$ has a generalized supplement 0 in $M$. Since $M=M_{1} \oplus M_{2}$, then $\operatorname{Rad}\left(M_{2}\right) \subseteq N+M_{1}$. It follows that $\operatorname{Rad}\left(M_{2}\right) \subseteq M_{2} \cap\left(N+M_{1}\right)$ has a generalized supplement $H$ in $M_{2}$ such that $H$ is a direct summand of $M_{2}$. By [10, Proposition 2.4], $H$ is a generalized supplement of $N+M_{1}$ in $M$. Moreover, $\operatorname{Rad}\left(M_{1}\right) \subseteq N+H$. Since $M_{1}$ is a generalized $\oplus$-radical supplemented module, $M_{1} \subseteq(N+H)$ has a generalized supplement $G$ in $M_{1}$ such that $G$ is a direct summand of $M_{1}$. Again applying [10, Proposition 2.4], $H+G$ is a generalized supplement of $N$ in $M$. It is clear that $H+G$ is a direct summand of $M$. Therefore, $M$ is a generalized $\oplus$-radical supplemented module.

(2) Let $U$ be any submodule such that $\operatorname{Rad}(M) \subseteq U$. Then $\operatorname{Rad}\left(M_{i}\right) \subseteq U \cap M_{i}$ for every $i \in I$. By this hypothesis, there exist submodules $V_{i}$ and $V_{i}^{\prime}$ of $M_{i}$ such that $M_{i}=\left(U \cap M_{i}\right)+V_{i}$, $\left(U \cap M_{i}\right) \cap V_{i} \subseteq \operatorname{Rad}\left(V_{i}\right)$, and $M_{i}=V_{i} \oplus V_{i}^{\prime}$ for every $i \in I$. Since $U$ is a fully invariant submodule of $M$, then $U=\oplus_{i \in I}\left(U \cap M_{i}\right)$. Let $V=\oplus_{i \in I} V_{i}$ and $V^{\prime}=\oplus_{i \in I} V_{i}^{\prime}$. Then there exist submodules $U$ and $V$ of $M$ such that $M=\oplus_{i \in I} M_{i}=\left[\oplus_{i \in I}\left(U \cap M_{i}\right)\right]+$ $\left(\oplus_{i \in I} V_{i}\right)=U+V, U \cap V=\oplus_{i \in I}\left(U \cap M_{i}\right) \cap\left(\oplus_{i \in I} V_{i}\right)=\oplus_{i \in I}[(U \cap$ $\left.\left.M_{i}\right) \cap V_{i}\right] \subseteq \oplus_{i \in I} \operatorname{Rad}\left(V_{i}\right)=\operatorname{Rad}(V)$ by [4, Theorem 21.6] and $V \oplus V^{\prime}=\left(\oplus_{i \in I} V_{i}\right) \oplus\left(\oplus_{i \in I} V_{i}^{\prime}\right)=\oplus_{i \in I} M_{i}=M$. Thus, $M$ is a generalized $\oplus$-radical supplemented module.

Proposition 6. If $M$ is a generalized $\oplus$-radical supplemented module, then $M / U$ is a generalized $\oplus$-radical supplemented module for every fully invariant submodule $U$ of $M$.

Proof. Let $U$ be any fully invariant submodule of $M$ and let $K / U$ be any submodule of $M / U$ with $\operatorname{Rad}(M / U) \subseteq K / U$. Since $(\operatorname{Rad}(M)+U) / U \subseteq \operatorname{Rad}(M / U)$, we have $\operatorname{Rad}(M) \subseteq K$. By the hypothesis, we have $M=K+V, K \cap V \subseteq \operatorname{Rad}(V)$, and $M=V \oplus V^{\prime}$ for some submodules $V$ and $V^{\prime}$ of $M$. By [11, Lemma 2.3] $(V+U) / U$ is generalized supplement of $K / U$ in $M / U$. Since $U$ is a fully invariant submodule of $M$, we have $U=(U \cap V)+\left(U \cap V^{\prime}\right)$ and $[(V+U) / U] \cap\left[\left(V^{\prime}+U\right) / U\right]=0$. Then $M / U=[(V+U) / U] \oplus\left[\left(V^{\prime}+U\right) / U\right]$. Hence, $M / U$ is a generalized $\oplus$-radical supplemented module.

Corollary 7. Let $R$ be a Dedekind domain and let $M$ be an $R$ module. $M$ is a generalized $\oplus$-radical supplemented module if and only if $M / P(M)$ is a generalized $\oplus$-radical supplemented module.

Proof. We know that $P(M)$ is a fully invariant submodule of $M$. So, by Proposition 6, $M / P(M)$ is a generalized $\oplus$-radical supplemented module. Conversely, suppose that $M / P(M)$ is a generalized $\oplus$-radical supplemented module. Since $R$ is a Dedekind domain, we have $M=P(M) \oplus N$ for some submodule $N$ of $M$. By the hypothesis, $N$ is a generalized $\oplus$-radical 
supplemented module. Hence, $M$ is a generalized $\oplus$-radical supplemented module by Theorem 5(1) and Corollary 3.

Example 8. Consider the left $\mathbb{Z}$-module $\mathbb{Q}$. According to Lemma 2, $\mathbb{Q}$ is a generalized $\oplus$-radical supplemented module. On the other hand, $\mathbb{Q}$ is not $\oplus$-supplemented because it is not torsion.

Theorem 9. Let $M$ be a module. Suppose that $\operatorname{Rad}(M)$ is small in $M$. Then $M$ is a generalized $\oplus$-radical supplemented module if and only if it is $\oplus$-supplemented.

Proof. $(\Rightarrow)$ Let $N$ be any submodule of $M$. Then $\operatorname{Rad}(M) \subseteq$ $\operatorname{Rad}(M)+N \subseteq M$. Since $M$ is a generalized $\oplus$-radical supplemented module, we have $M=\operatorname{Rad}(M)+N+L$, $(\operatorname{Rad}(M)+N) \cap L \subseteq \operatorname{Rad}(L)$, and $M=L \oplus L^{\prime}$ for submodules $L, L^{\prime} \leq M$. Since $\operatorname{Rad}(M) \ll M$, we have $M=N+L$, $N \cap L \ll L$. Therefore $M$ is $\oplus$-supplemented.

$(\Leftarrow)$ is trivial.

Corollary 10. Every generalized $\oplus$-radical supplemented module over a left Bass ring is $\oplus$-supplemented.

Proposition 11. Every direct summand of $P(M)$ is a generalized $\oplus$-radical supplemented module for every $R$-module $M$.

Proof. Let $N$ be any direct summand of $P(M)$. Then there exists a submodule $L$ of $P(M)$ such that $P(M)=N \oplus L$. Since $P(M)=\operatorname{Rad}(P(M))=\operatorname{Rad}(N) \oplus \operatorname{Rad}(L)$ by [4, Theorem 21.6], then $N=\operatorname{Rad}(N) \oplus[N \cap \operatorname{Rad}(L)]=\operatorname{Rad}(N)$. By Lemma 2, $N$ is generalized $\oplus$-radical supplemented.

Proposition 12. Let $M$ be a generalized $\oplus$-radical supplemented module. Suppose that a cofinite fully invariant submodule $K$ of $M$ is a direct summand of $M$. Then $K$ is generalized $\oplus$-radical supplemented.

Proof. Let $U$ be any submodule of $K$ with $\operatorname{Rad}(K) \subseteq U$. By the hypothesis, we have $M=K \oplus L$ for some finitely generated submodule $L$ of $M$. Then $\operatorname{Rad}(L) \ll L$. Clearly $\operatorname{Rad}(M) \subseteq$ $U+\operatorname{Rad}(L)$. So there exist submodules $V$ and $V^{\prime}$ of $M$ such that $M=U+\operatorname{Rad}(L)+V,(U+\operatorname{Rad}(L)) \cap V \subseteq \operatorname{Rad}(V)$, and $M=V \oplus V^{\prime}$. Since $\operatorname{Rad}(L) \ll L$, we have $M=U+V$, $U \cap V \subseteq \operatorname{Rad}(V)$ and $M=V \oplus V^{\prime}$. It follows that $K=U+(K \cap$ $V)$ and $U \cap(K \cap V) \subseteq \operatorname{Rad}(M)$. Since $K$ is a fully invariant submodule of $M$, then $K=(K \cap V) \oplus\left(K \cap V^{\prime}\right)$. Note that $U \cap(K \cap V) \subseteq \operatorname{Rad}(K \cap V)$. Therefore, $K$ is generalized $\oplus$ radical supplemented.

Lemma 13. Let $M$ be a module and $N$ a submodule such that $\operatorname{Rad}(M) \subseteq N$. If $N$ is a direct summand of $M$, then $\operatorname{Rad}(M)=\operatorname{Rad}(N)$. In particular, if $\operatorname{Rad}(M)$ is a direct summand of $M$, then $\operatorname{Rad}(M)=P(M)$.

Proof. Let $N$ be any direct summand of $M$ such that $\operatorname{Rad}(M) \subseteq N$. Then there exists a submodule $N^{\prime}$ of $M$ such that $M=N \oplus N^{\prime}$. By [4, Theorem 21.6], $\operatorname{Rad}(M)=$ $\operatorname{Rad}(N) \oplus \operatorname{Rad}\left(N^{\prime}\right)$. Since $\operatorname{Rad}(M) \subseteq N, \operatorname{Rad}(M)=$ $\left[\operatorname{Rad}(N) \oplus \operatorname{Rad}\left(N^{\prime}\right)\right] \cap N=\operatorname{Rad}(N) \oplus\left[\operatorname{Rad}\left(N^{\prime}\right) \cap N\right]=$ $\operatorname{Rad}(N)$. Now we take $N=\operatorname{Rad}(M)$ under the similar condition. $\operatorname{Rad}(M)=\operatorname{Rad}(\operatorname{Rad}(M))$; that is, $\operatorname{Rad}(M)$ is radical. Consequently, $\operatorname{Rad}(M)=P(M)$.

Let $R$ be a ring and let $M$ be an $R$-module. We consider the following condition.

$\left(\mathrm{D}_{3}\right)$ If $M_{1}$ and $M_{2}$ are direct summands of $M$ with $M=$ $M_{1}+M_{2}$, then $M_{1} \cap M_{2}$ is also a direct summand of $M$.

Proposition 14. Let $M$ be a generalized $\oplus$-radical supplemented module with $\left(D_{3}\right)$ and let $N$ be a submodule with $\operatorname{Rad}(M) \subseteq N$. If $N$ is a direct summand of $M, N$ is generalized $\oplus$-radical supplemented.

Proof. Let $U$ be a submodule of $N$ such that $\operatorname{Rad}(N) \subseteq U$. By Lemma 13, $\operatorname{Rad}(M)=\operatorname{Rad}(N)$. Since $M$ is a generalized $\oplus$-radical supplemented module, there exist submodules $V$ and $V^{\prime}$ of $M$ such that $M=U+V, U \cap V \subseteq \operatorname{Rad}(V)$, and $M=V \oplus V^{\prime}$. Then $N=U+(N \cap V)$. Since $M$ satisfies $\left(\mathrm{D}_{3}\right), N \cap V$ is a direct summand of $M$. Then there exists a submodule $X$ of $M$ such that $M=(N \cap V) \oplus X$. It follows that $U \cap(N \cap V) \subseteq \operatorname{Rad}(N \cap V)$ and $N=(N \cap V) \oplus(N \cap X)$. Therefore $N$ is a generalized $\oplus$-radical supplemented module.

A module $M$ is called local if $M / \operatorname{Rad}(M)$ is simple and $\operatorname{Rad}(M)$ is small in $M$ [10]. The module $M$ is called $w$-local if $M / \operatorname{Rad}(M)$ is simple [12].

The following fact is clear.

Corollary 15. Every $w$-local module is generalized $\oplus$-radical supplemented.

Lemma 16. Let $M$ be an indecomposable module. If $M$ is a generalized $\oplus$-radical supplemented module, then $M=$ $\operatorname{Rad}(M)$ or $M$ is $w$-local.

Proof. Suppose that $\operatorname{Rad}(M) \neq M$. Then $M$ contains at least one maximal submodule, say $K$. Since $\operatorname{Rad}(M) \subseteq K$, then there exist submodules $V$ and $V^{\prime}$ of $M$ such that $M=K+V$, $K \cap V \subseteq \operatorname{Rad}(V)$, and $M=V \oplus V^{\prime}$. We have $V=M$ because $M$ is indecomposable. Since $V$ is a generalized supplement of $K, V$ is $w$-local by [12, Lemma 3.3]. So $M$ is $w$-local.

Theorem 17. Let $R$ be a local commutative ring and $M$ be a uniform R-module. Every submodule of $M$ is generalized $\oplus$ radical supplemented if and only if it is uniserial.

Proof. $(\Rightarrow)$ By [13, Lemma 6.2], it suffices to show that every finitely generated submodule of $M$ is local. Let $N$ be any finitely generated submodule of $M$. By assumption, $N$ is indecomposable. So, by Lemma $16, N$ is $w$-local. Since $N$ is finitely generated, $N$ is local.

$(\Leftarrow)$ Since $M$ is uniserial, every submodule of $M$ is hollow by $[3,2.17]$. So it is easy to see that every submodule of $M$ is a generalized $\oplus$-radical supplemented module.

Corollary 18. Let $R$ be a local commutative ring. Suppose that every submodule of $E(R / \operatorname{Rad}(R))$ is generalized $\oplus$-radical supplemented, where $E(R / \operatorname{Rad}(R))$ is the injective hull of the simple module $R / \operatorname{Rad}(R)$. Then $R$ is a uniserial ring. 
Proof. Since $E(R / \operatorname{Rad}(R))$ is uniform, the hypothesis implies that $E(R / \operatorname{Rad}(R))$ is uniserial by Theorem 17. It follows from $[13$, Lemma 6.2] that $R$ is a uniserial ring.

Theorem 19. The following statements are equivalent for a ring $R$.

(1) $R$ is semiperfect.

(2) Every finitely generated free $R$-module is generalized $\oplus$ radical supplemented.

Proof. By [14, Theorem 2.1] and Theorem 9, the proof is clear.

Lemma 20. Let $M$ be a module. Suppose that $\operatorname{Rad}(M)=0$. Then $M$ is a generalized $\oplus$-radical supplemented module if and only if it is semisimple.

Proof. This is clear by [15, Proposition 3.3].

A ring $R$ is called left $V$-ring if every simple left $R$-module is injective. The ring $R$ is called an SSI ring if semisimple left $R$-module is injective. Let $R$ be a commutative ring. $R$ is regular if and only if every simple left $R$-module is injective [13, Theorem 2.14].

Proposition 21. Let $R$ be a left $V$-ring and $M$ an $R$-module. Then $M$ is generalized $\oplus$-radical supplemented if and only if $M$ is semisimple.

Corollary 22. Let $R$ be a commutative regular ring and $M$ an $R$-module. Then $M$ is generalized $\oplus$-radical supplemented if and only if $M$ is semisimple.

Proposition 23. The following statements are equivalent for a $\operatorname{ring} R$.

(1) Every generalized $\oplus$-radical supplemented $R$-module is injective.

(2) $R$ is left Noetherian $V$-ring.

Proof. By [16, Proposition 5.3], the proof is clear.

\section{Conflict of Interests}

The authors declare that there is no conflict of interests regarding the publication of this paper.

\section{References}

[1] F. Kasch, Modules and Rings, vol. 17, Academic Press, London, UK, 1982.

[2] A. V. Kelarev, Ring Constructions and Applications, vol. 9, World Scientific, River Edge, NJ, USA, 2002.

[3] J. Clark, C. Lomp, N. Vanaja, and R. Wisbauer, Lifting Modules. Supplements and Projectivity in Module Theory, Frontiers in Mathematics, Birkhäuser, Basel, Switzerland, 2006.

[4] R. Wisbauer, Foundations of Module and Ring Theory, vol. 3, Gordon and Breach, Philadelphia, Pa, USA, 1991.
[5] S. H. Mohamed and B. J. Müller, Continuous and Discrete Modules, vol. 147 of London Mathematical Society Lecture Note, Cambridge University Press, Cambridge, UK, 1990.

[6] H. Zöschinger, "Moduln, die in jeder Erweiterung ein Komplement haben," Mathematica Scandinavica, vol. 35, pp. 267-287, 1974.

[7] W. Xue, "Characterizations of semiperfect and perfect rings," Publicacions Matemàtiques, vol. 40, no. 1, pp. 115-125, 1996.

[8] H. Çalışıcı and E. Türkmen, "Generalized $\oplus$-supplemented modules," Algebra and Discrete Mathematics, vol. 10, no. 2, pp. 10-18, 2010.

[9] A. Idelhadj and R. Tribak, "On some properties of $\oplus$ supplemented modules," International Journal of Mathematics and Mathematical Sciences, no. 69, pp. 4373-4387, 2003.

[10] Y. Wang and N. Ding, "Generalized supplemented modules," Taiwanese Journal of Mathematics, vol. 10, no. 6, pp. 1589-1601, 2006.

[11] E. Türkmen and A. Pancar, "On cofinitely Rad-supplemented modules," International Journal of Pure and Applied Mathematics, vol. 53, no. 2, pp. 153-162, 2009.

[12] E. Büyükașik and C. Lomp, "On a recent generalization of semiperfect rings," Bulletin of the Australian Mathematical Society, vol. 78, no. 2, pp. 317-325, 2008.

[13] D. W. Sharpe and P. Vámos, Injective Modules, Cambridge University Press, Cambridge, UK, 1972.

[14] D. Keskin, P. F. Smith, and W. Xue, "Rings whose modules are $\oplus$ supplemented modules," Acta Mathematica Hungarica, vol. 83, pp. 161-169, 1999.

[15] K. Varadarajan, "Dual Goldie dimension," Communications in Algebra, vol. 7, no. 6, pp. 565-610, 1979.

[16] C. Lomp, "On semilocal modules and rings," Communications in Algebra, vol. 27, no. 4, pp. 1921-1935, 1999. 


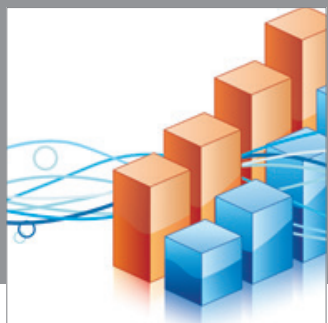

Advances in

Operations Research

mansans

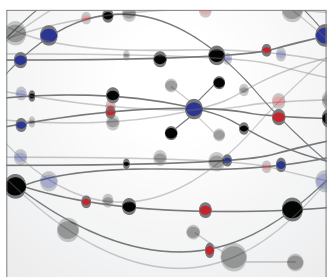

The Scientific World Journal

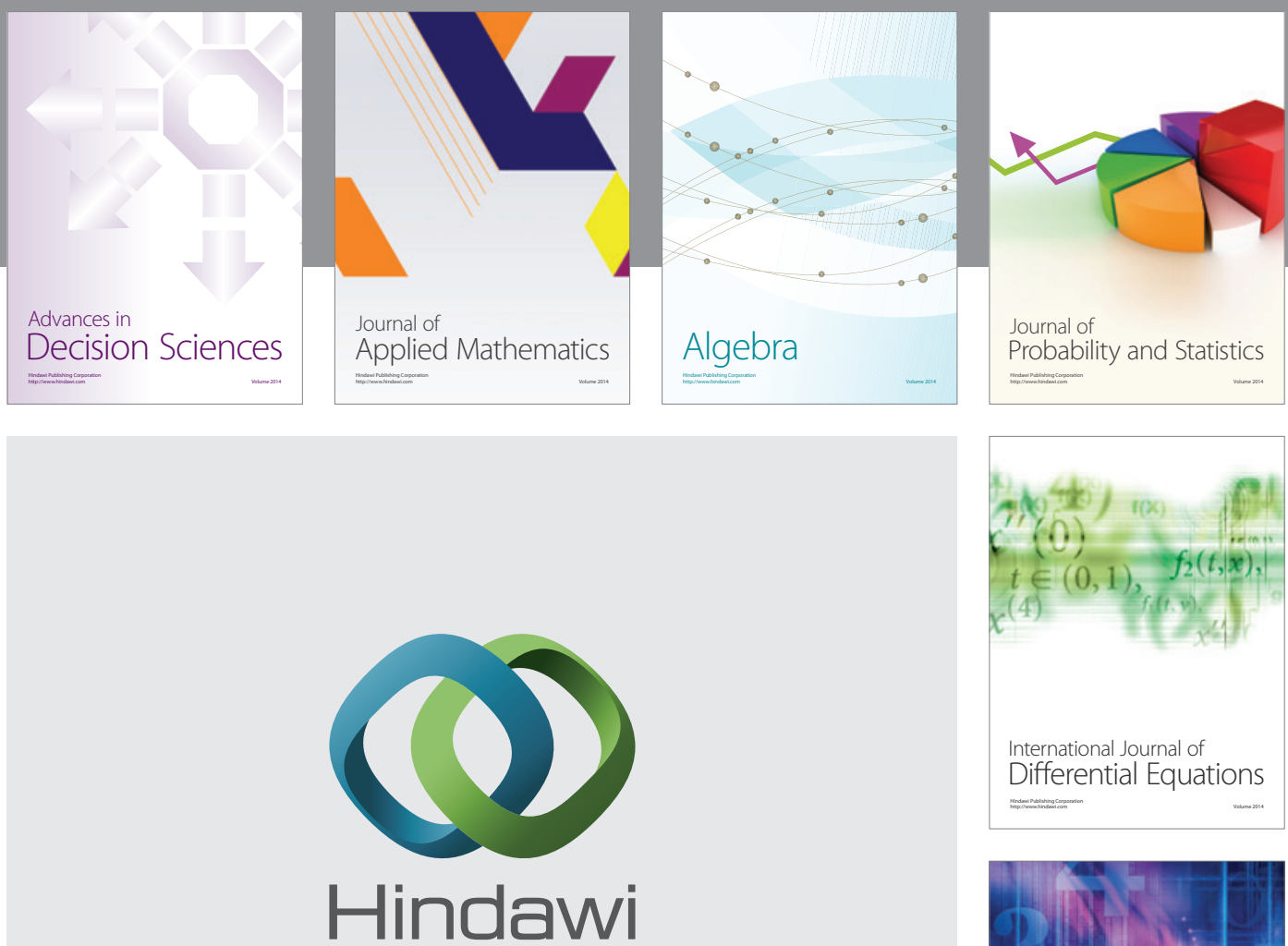

Submit your manuscripts at http://www.hindawi.com
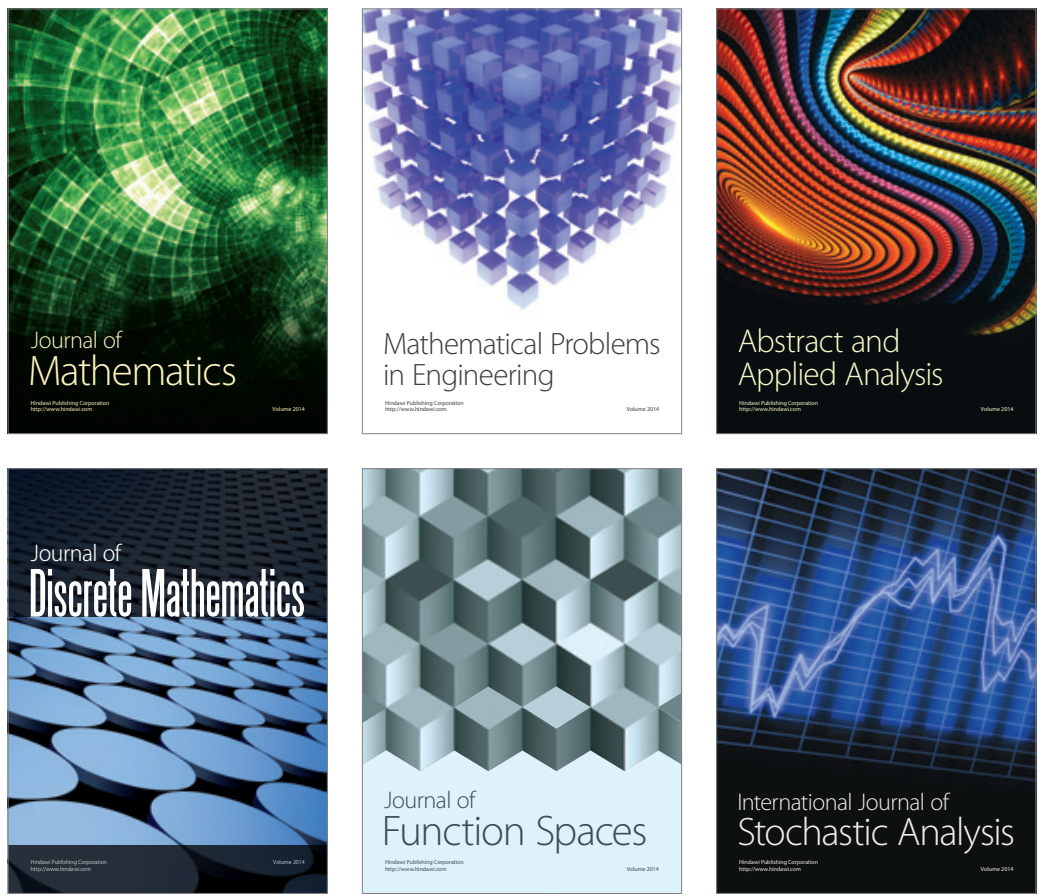

Journal of

Function Spaces

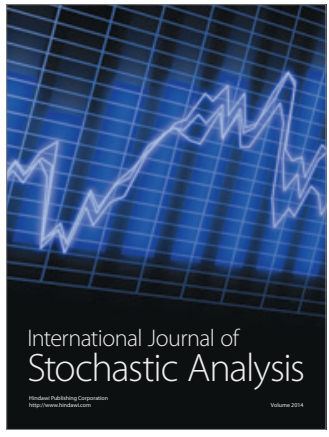

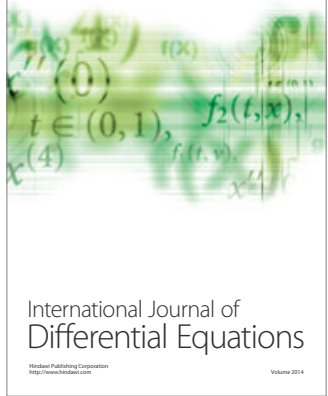
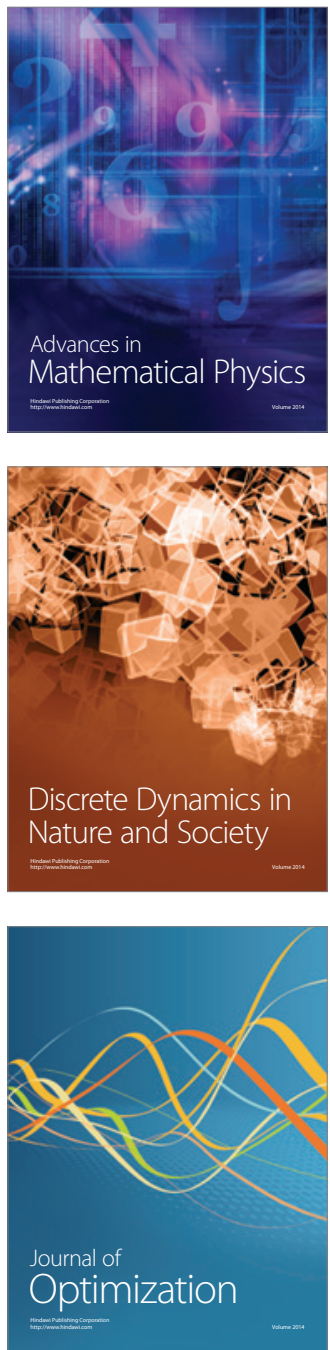